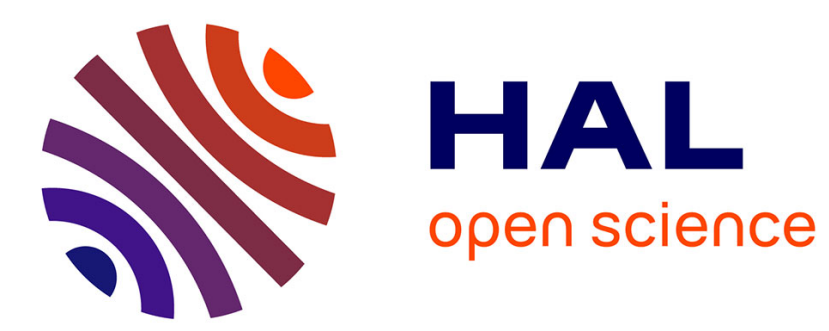

\title{
OUTDOOR PROPAGATION OF FINITE-AMPLITUDE NOISE
}

\author{
A. Webster, D. Alexander, D. Blackstock
}

\section{To cite this version:}

A. Webster, D. Alexander, D. Blackstock. OUTDOOR PROPAGATION OF FINITE-AMPLITUDE NOISE. Journal de Physique Colloques, 1979, 40 (C8), pp.C8-325-C8-332. 10.1051/jphyscol:1979858 . jpa-00219564

\section{HAL Id: jpa-00219564 https://hal.science/jpa-00219564}

Submitted on 1 Jan 1979

HAL is a multi-disciplinary open access archive for the deposit and dissemination of scientific research documents, whether they are published or not. The documents may come from teaching and research institutions in France or abroad, or from public or private research centers.
L'archive ouverte pluridisciplinaire HAL, est destinée au dépôt et à la diffusion de documents scientifiques de niveau recherche, publiés ou non, émanant des établissements d'enseignement et de recherche français ou étrangers, des laboratoires publics ou privés. 


\title{
OUTDOOR PROPAGATION OF FINITE-AMPLITUDE NOISE
}

\author{
WEBSTER A. - ALEXANDER D.E. and BLACKSTOCK D.T.
}

Applied Research Laboratories - The University of Texas at Austin

Austin, Texas 78712 USA

Résumé. - Une étude expérimentale sur un bruit d'amplitude finie a été réalisêe en extérieur.

La source utilisée est un arrangement de cornets en verticale ascendante pour éviter les effets du sol. Des spectres ont été relevés sur T'axe de propagation verticale jusqu'à une distance de $80 \mathrm{~m}$. La plupart des essais cnt été conduits avec une bande de bruit de 1 octave dont les fréquences cen trales variaient de 2 à $10 \mathrm{kHz}$ et les amplitudes de 121 à $145 \mathrm{~dB}$ ( $r . e .20 \mu \mathrm{Pa}$ à $1 \mathrm{~m}$ ). Les résultats expérimentaux ont été comparés aux prédictions de la théorie linéaire. Les conclusions essentielles sont :

1. Aux amplitudes les plus fortes, la distorsion par propagation non linéaire entraine une forte génération de bruit haute fréquence sur toute la distance de propagation. Il existe une fajble génération de bruit à basse fréquence.

2. Cette gênération existe aussi bien dans le champ proche que dans le champ lointain. La gênëration haute fréquence dans le champ proche est même supérieure aux effets de la diffraction.

3. En aucun point de mesure, on n'a pu établir un comportement de petit signal pour le bruit haute fréquence.

4. Avec une échelle appropriêe, les spectres observês sont analogues à ceux des bruits de réacteurs. Il est donc à peu près certain que cette sorte de distorsion non linéaire affecte également le bruit des reacteurs actuels.

Abstract. - Experiments on the propagation of finite-amplitude noise outdoors have been made. The source was an array of horns pointed upward to avoid ground effects. Spectra were recorded along the vertical propagation path at distances out to $80 \mathrm{~m}$. In most of the experiments the source emitted noise approximately one octave in bandwidth. The frequency range was 2 to $10 \mathrm{kHz}$ and the source level range was 121 to $145 \mathrm{~dB}$ (overa11) re $20 \mu \mathrm{Pa}$ at $1 \mathrm{~m}$. Measurements were compared with predictions based on linear theory. Major conclusions are as follows :

1. At the higher source levels nonlinear propagation distorsion caused a strong generation of high frequency noise over the entire propagation path. There was little generation of low frequency noise.

2. The generation occured in both the nearfield and the farfield of the source. Nearfield generation exceeded the high-frequency boost caused by diffraction.

3. At no measurement point was small-signal behavior established for the high-frequency noise.

4. Approprietely scaled, the measured spectra were found to be well within the jet noise range. Therefore, nonlinear distortion of the sort we observed probably affects actual jet noise as well.

1. Introduction. - Experiments have been done out doors on the propagation of intense acoustic noise generated by a conventional electroacoustic source. These experiments constitute the second of a research program begum in 1975 to study outdoor propagation of finite-amplitude sound waves. The ultimate application of the research is to jet aircraft noise. In particular, the goal was do determine whether aircraft noise is affected by nonlinear propagation distortion. The experiments were carried out along a vertical propagation path in order to avoid grounf effects. The path, about $80 \mathrm{~m}$ in length was parallel to a radio tower, whose elevator car ried the microphone. An initial set of experiments done with intense tones $/ 1,2 /$ was reported at the 9 th International Congress on Acoustics (Madrid, (1977) /3/. Important nonlinear effects were observed : harmonic distortion, skock formation, extra attenuation, and an interaction between diffraction and distortion. Experiments with intense noise were then undertaken; the results are reported here. A more complete description is given in a NASA report/4/, which also contains a summary of the tone experiments.

Previous investigations of finite-amplitude acoustic noise have been largely limited to plane waves in tubes $/ 5,6 /$ or to strictly theoretical calculations (see, for example, Refs. 7 and 8 ). 
EXPERIMENTAL
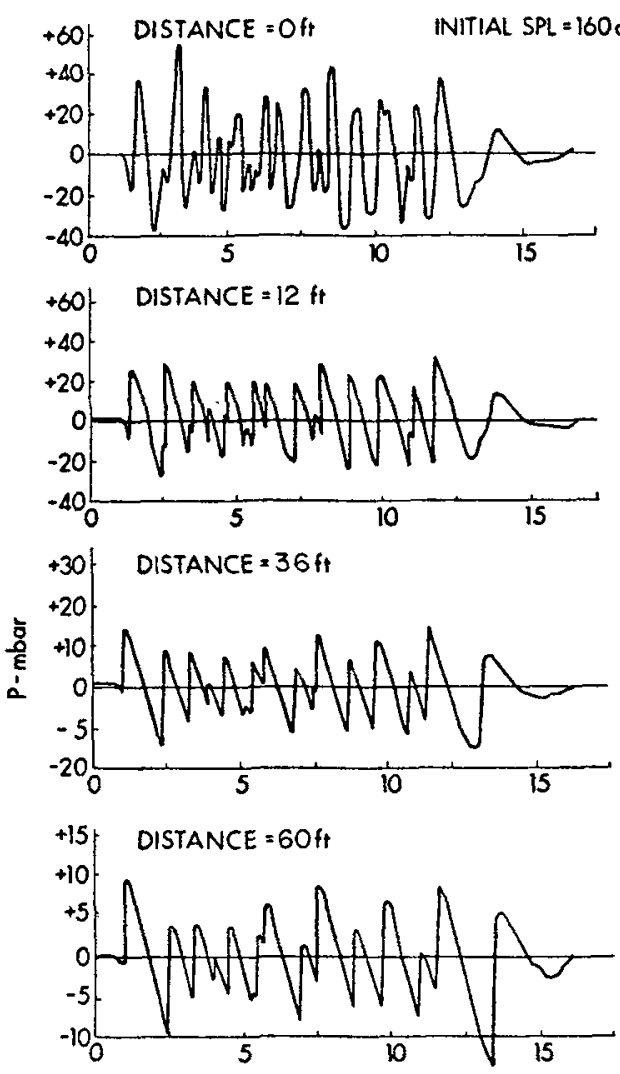

+15 DISTANCE $=84 \mathrm{fr}$

$+10$

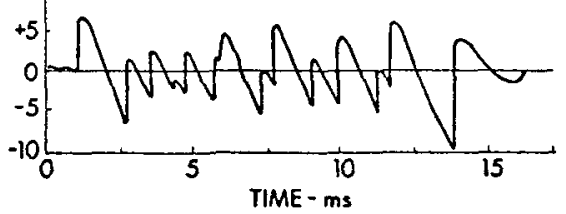

COMPUTED
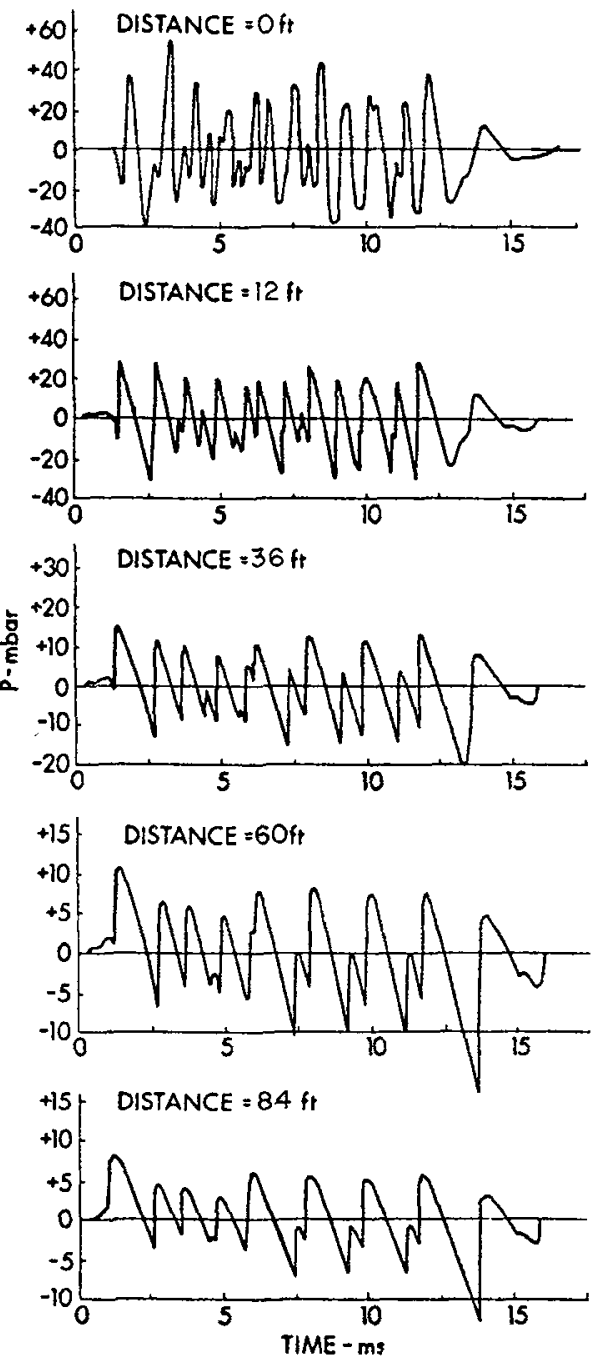

Fig. 1. - Measured and computed waveforms for a broadband noise pulse Taken from ref. 6 .

The present work is an outgrowth of Pestorius's tube experiments /6/, an example of which is shown in Fig. 1. The figure showns a sequence of time waveforms (measured on the left, computed on the right) of a very intense noise pulse as it propagates down a plane wave tube. Two different stages of distortion may be identified. In Stage I many shocks form as peaks overtake troughs. Because of the very high intensity of this particu lar noise, Stage I is already complete at the 12 ft measurement point. Thereafter, in Stage II, the distortion is dominated by shock merging (the big shocks tend to "eat up" the little shocks), which is a relatively slow process. The noise waveform becomes simpler in appearance because the number of zero crossings is reduced. The spectral changes associated with the two stages are very different. Stage I is marked by a strong growth of the highfrequency end of the spectrum, Stage II by a growth of the low-frequency end. of course, there is a corresponding reduction of the intense, middle part of the spectrum, but the reduction is most noticeable during Stage II, when shock dissipation is very strong.

Three major changes are faced when the finiteamplitude noise experiment is moved outdoors. First and most important is the spherical spreading of the waves, which causes a dramatic slowing down of the distortion process. Moreover, because of the long distance required for shocks to form, ordinary 
absorption in the medium becomes very important. The acoustic source must therefore be extremely powerful if Stage II in the distortion process is to be reached. We observed only stage I distortion in our experiments. Second, diffraction must be contented with because, as a matter of practical necessity, directional sources must be used. Because we do not understand the interaction of diffraction and nonlinear distortion very well, quantitative interpretation of the data is difficult. Finally, wind and temperature fluctuations make the outdoor medium randomly inhomogeneous. We have found, however, that the fluctuations have only a mild effect on distortion. In the tone experiments $/ 1 /$, for example, although random inhomogeneity definitely affected the instantaneous time waveform of the received signal, it had little or no residual effect on the long term average spectral content of the signal. In fact, long term average measurements compared very wel1 with theoretical predictions obtained by assuming the medium homogeneous.
2. Experimental Apparatus and Procedure. - A general schematic of the experiment is shown in Fig. 2 . The source, mounted on a table that could be tilted and/ or rotated to allow alignment and measurement of beam patterns, was an array of exponential horns. one array (AEM) was composed of 20 square-mouth horns joined at the mouths to form a $45.7 \mathrm{~cm} \times 57.2$ $\mathrm{cm}$ rectangular aperture. The other array (JBL) was composed of 1, 3, 7, or 10 circular horns (mouth diameter $15.5 \mathrm{~cm}$ ) closely packed to have either a circular or triangular aperture. The propagation distance was varied by moving the elevator car, which carried the traveling microphone ( $B \& K$ 4136), up or down the tower.

Eleven noise experiments were completed. Each experiment consisted of choosing a source spectrum and source level and measuring the transmitted sprectrum at various axial distances $r$ from the array aperture. Source level, denoted $\mathrm{OASPL}_{1 \mathrm{~m}}$, is overa 11 sound pressure leve 1 ( $\mathrm{dB}$ re $20 \mu \mathrm{Pa}$ ) based on a far-

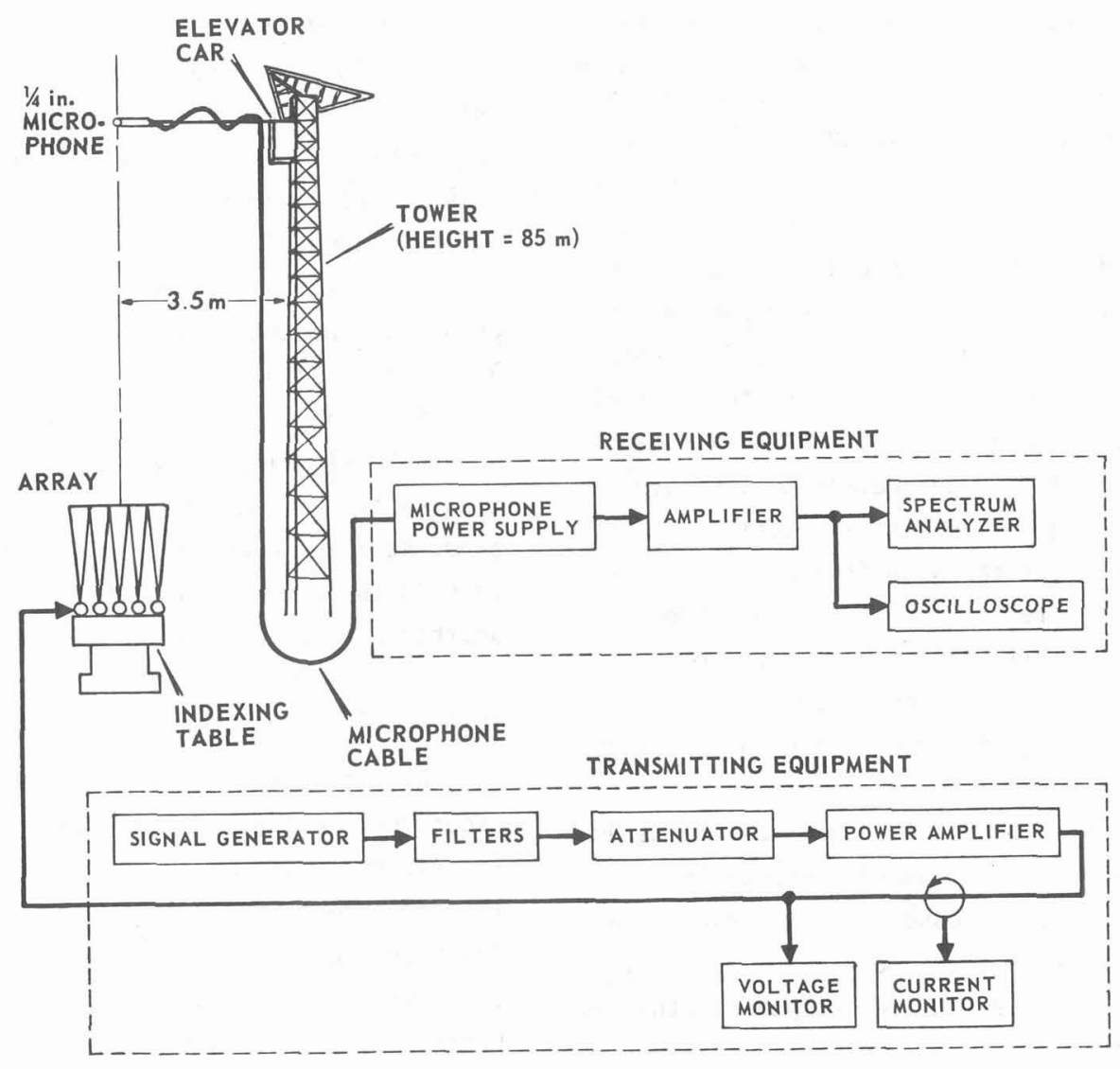

Fig. 2. - Block diagram of experimental arrangement. 
field measurement but extrapolated back to the stan dard distance $r=1 \mathrm{~m}$. The bandwidth of the electrical imput to the source was usually about an octave (3-octave and 1/3-octave bands were also used). The meteorological conditions (ground leve1) were as follows : temperature range 23 to $31^{\circ} \mathrm{C}$, relative humidity range 55 to $90 \%$, and wind speed range 0 to $24 \mathrm{~km} / \mathrm{h}$.

\section{3. - Experimental Results ans Comparison with Linear theory.}

Data from of the experiments, JBL5 and AEM4, are presented in this section. First, however, the predictions against which the data were compared are discussed.

The simplest way to determine whether propagating noise is subject to nonlinear distortion is to compare the data with predictions based on linear theory. what should be included in the linear theory model depends on the complexities of the propagation path being considered. If all measurements are in the farfield, only spherical spreading and atmospheric atmospheric attenuation /9/ need be accounted for. If nearfield measurements are included, piston radiation theory may be used to pass from the spectrum at the aperture to that in the farfield. The farfield begins at approximately the Rayleigh distance $R_{0}=S / \lambda$, where $S$ is the aperture area and $\lambda$ is the wavelength. Note that because $R_{0}$ is proportional to frequency, the low-frequency part of the spectrum begins to spread spherically before the high-frequency part does. As a result, the farfield spectrum of the noise acquires a $6 \mathrm{~dB} /$ octave highfrequency boost relative to the aperture spectrum. This spectral change is due to diffraction and must be distinguished from the high frequency boost caused by nonlinear effects. Finally, if measurements inside the array, that is, inside one of the horns, are included, conventional horn theory may be used to compute the propagation from the horn throat to the horn mouth.

Figure 3 shows the results for Experiments JBL5. A 7-horn circular array produced a source level of $140 \mathrm{~dB}$. The ordinate is sound pressure level in a $300 \mathrm{~Hz}$ band. All measurement points are in the far. field, that is, $R_{0}<9.14 \mathrm{~m}$ for components that made up the aperture spectrum. The electrical imput signal to the array was an octave band of noise centered at $4 \mathrm{kHz}$. It is clear that much distortion occured before the $9.14 \mathrm{~m}$ distance was reached. The initial linear theory spectrum is a copy of the $9.14 \mathrm{~m}$ measured spectrum (except that the anomalous upturn at very low frequency is not reproduced). The linear theory predictions work well for the original octave band but poorly for the high frequency noise. For example, the measured spectrum exceeds the linear theory prediction at $r=79.3 \mathrm{~m}$ by $4 \mathrm{~dB}$ at $10 \mathrm{kHz}, 15 \mathrm{~dB}$ at $20 \mathrm{kHz}, 30 \mathrm{~dB}$ at $30 \mathrm{kHz}$, and $40 \mathrm{~dB}$ at $35 \mathrm{kHz}$ (above $35 \mathrm{kHz}$ the measured spectrum is contaminated by electrical and/ or ambient acoustical noise). At a given frequency the discrepancy increases with range. For example, at $25 \mathrm{kHz}$ the discrepancy is about $5 \mathrm{~dB}$ at $18.3 \mathrm{~m}, 10 \mathrm{~dB}$ at $36.6 \mathrm{~m}$, and $20 \mathrm{~dB}$ at $79.3 \mathrm{~m}$. There is apparently no tendency for the discrepancy to reach a plateau, at least over the measurement range available to us.

Experiment AEM4 (Fig. 4.) consisted of two sets of measurements, one about $20 \mathrm{~dB}$ higher in level than the other. The spectral shape of the input noise was the same for both sets. At the first measurement point, which was inside one of the horns $(r=-0.23 \mathrm{~m})$, the OASPL was about $126 \mathrm{~dB}$ for the high level noise. Even for the low level noise, nonlinear propagation distorsion was not negligible. There is a clearcut growth of the second harmonic band and even a development of a third harmonic band. The high level data show a much greater effect of nonlinearity. There is a robust growth of the entire spectrum above the original $4 \mathrm{kHz}$ band. Even at $20 \mathrm{kHz}$, where atmospheric absorption is strong, the growth is impressive. Inside the horns the signal at $20 \mathrm{kHz}$ is in the instrumentation noise, at least $60 \mathrm{~dB}$ below the fundamental band. At $70 \mathrm{~m}$, however, the $20 \mathrm{kHz}$ signal is within $30 \mathrm{~dB}$ of the fundamental band. To put it another another way, the $20 \mathrm{kHz}$ level is about the same at $70 \mathrm{~m}$ at is seems to be at $0 \mathrm{~m}$. A sma 11 but notice able low frequencygrowth may also be discerned.

Figure 5 shows a comparison of data from Experiment AEM4 with predictions besed on linear theory. The starting point for each prediction is the spectrum measured inside the horn $(r=-0.23 \mathrm{~m})$. The high-frequency boost caused by diffraction is therefore included in the predicted spectra. This boost clearly does not account for the high-frequency growth that actually takes place, even for the low level measurements. As was the case for Experiment JBL5 (Fig. 3), linear theory provides 


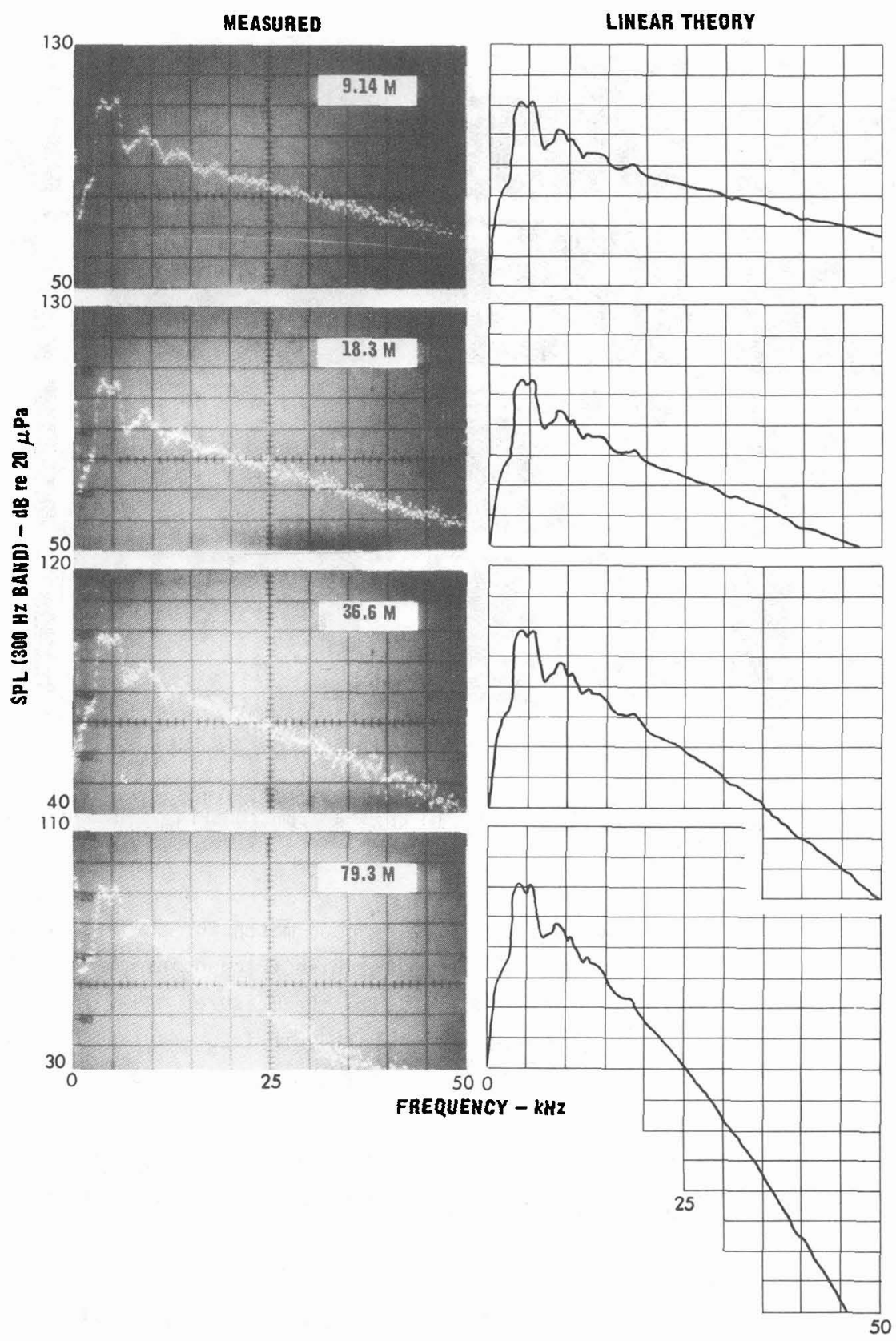

Fig. 3 - Experiment JBL5. Seven-horn array, $R_{0}(20 \mathrm{kHz})=7.42 \mathrm{~m}, O A S P L_{1 m}=140 \mathrm{~dB}$. Electrical input was an octave band centered at $4 \mathrm{kHz}$. Linear theory predictions are based on spherical spreading and atmospheric attenuation only. 

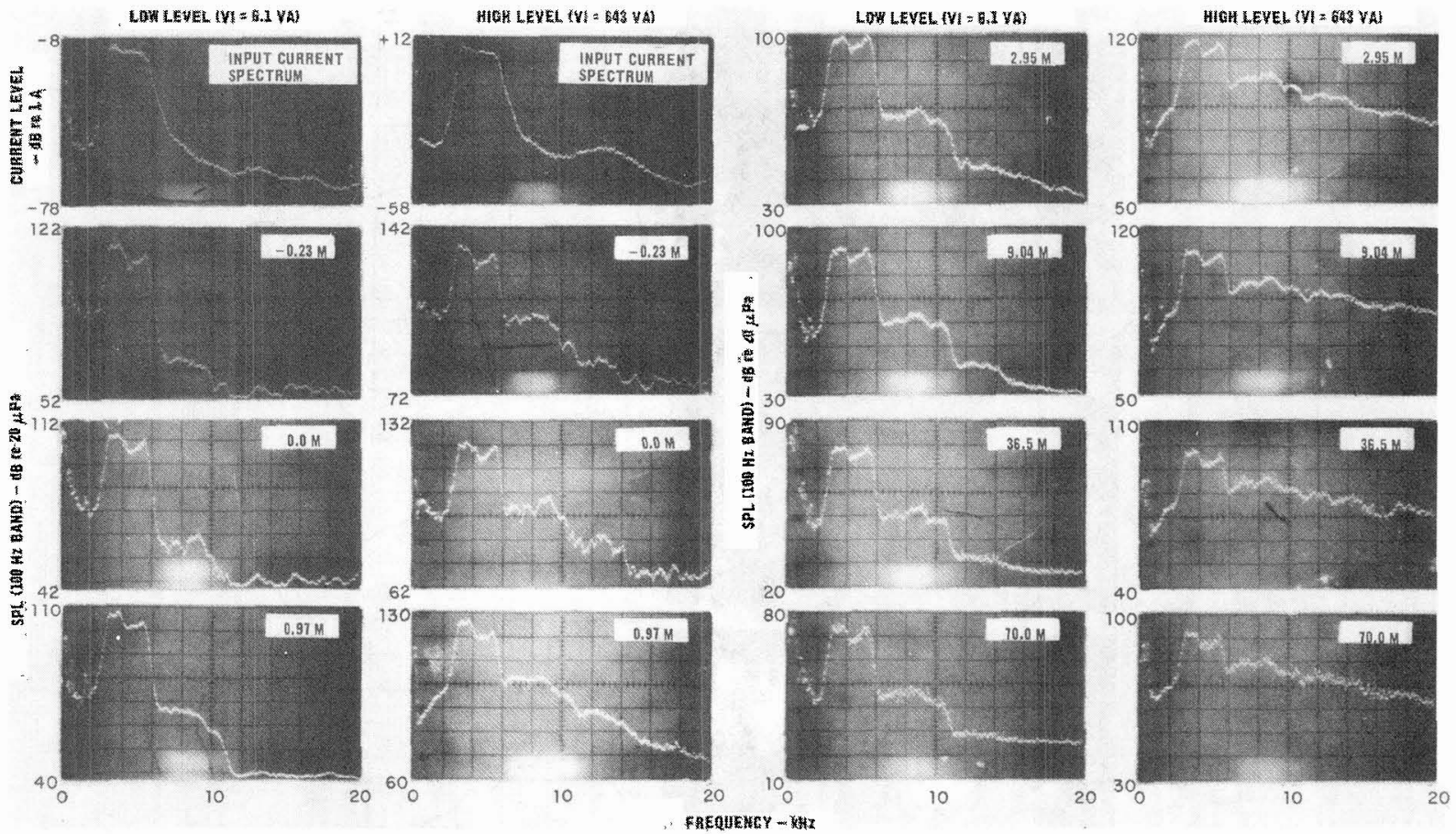

Fig. 4. - Experiment AEM4, twenty-horn array, $\mathrm{R}_{0}(10 \mathrm{kHz})=7.6 \mathrm{~m}, 0 \mathrm{ASPL}{ }_{1 \mathrm{~m}}=123 \mathrm{~dB}$ (low level data), $\mathrm{OASPL}_{\text {Im }}=142 \mathrm{~dB}$ (high level data).

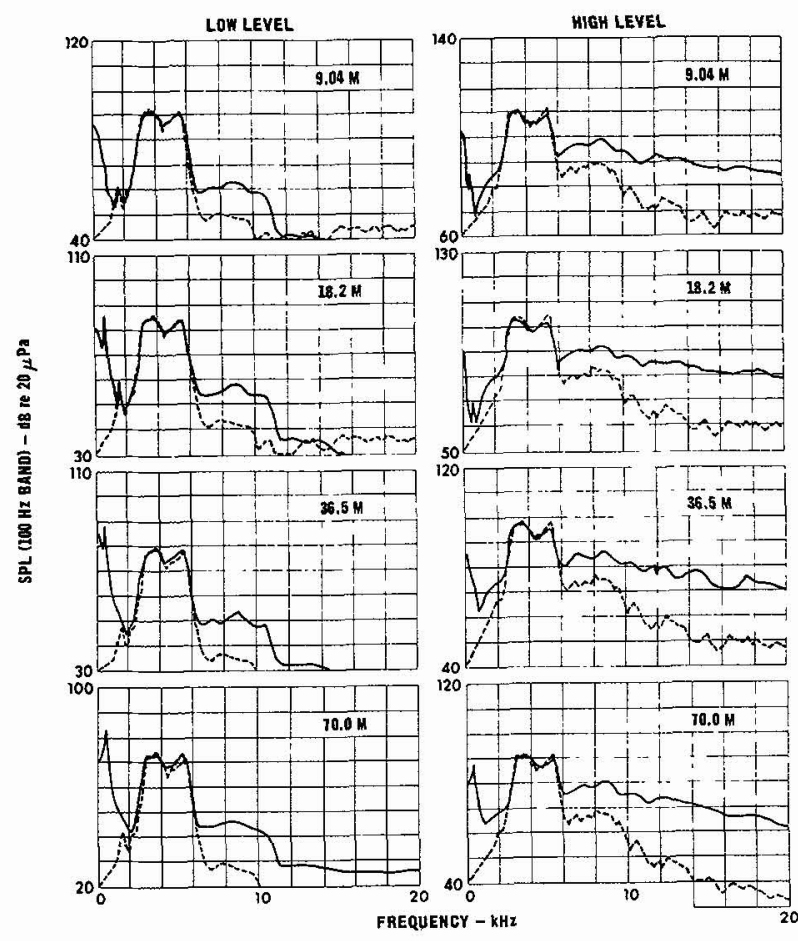

Fig.5. - Experiment AEM4, comparison with 1 inear theory. Solid curves : measurements. Dashed curves : linear theory based on spectra measured at $r=-0.23 \mathrm{~m}$ (see Fig. 4). a good match for the data in the initial band, but at high frequencies the predicted spectra fall blow the measured ones. The discrepancy increases with both frequency and distance. This effect was noticed in all the other experiments as well.

4. Discussion and Conclusions. - Two points are discussed in detail in this section, the behavior of the high frequency noise components and the application of the research to aircraft noise.

The character of the high-frequency discrepancy between measurements and linear theory predictions is of considerable interest. The fact that the discrepancy continues to grow with distance implies that the high-frequency noise has no "small-signal farfield," that is, there is no region where it is free of nonlinear effects. There is firm theoretical support for this conclusion. Consider, for example, the propagation of a plane periodic wave of finite amplitude in an absorbing medium. If $2 \alpha_{1}<\alpha_{2}$, the (nonlinearly generated) second harmonic component is found to decay asymp totically as $\exp \left(-2 \alpha_{1} x\right)$, not, as one would expect from linear theory, $\exp \left(-\alpha_{2} x\right)$. Here $\alpha_{1}$ and $\alpha_{2}$ are the small-signal attenuation coefficients at the 
fundamental and second harmonic frequencies, respectively, and $x$ is distance from the source. The second harmonic therefore decays more slowly than one would expect from linear theory. Moreover, no distance is ever reached at which the small-signal decay, exp $\left(-\alpha_{2} x\right)$, takes over. If the wave is spherical, the asymptotic decay is found to be $r^{-2} \exp \left(-2 \alpha_{1} r\right)$. Similarly the third harmonic decays asymptotically as $r^{-3} \exp \left(-3 \alpha_{1} r\right)$, and so on $/ 4 /$. This behavior, which is certainly foreign to linear theory, is a result of the way the higher frequency components are generated. They are produced not by the source but by nonlinear interaction that takes place over the entire travel path between source and observation point.

To determine the relevance of our results to actual jet aircraft noise, we have compared spectra from two of our experiments with those produced by a KC-135A aircraft (ground runup, single engine, measurement angle $30^{\circ}$ off the jet axis). In Fiq. 6 .

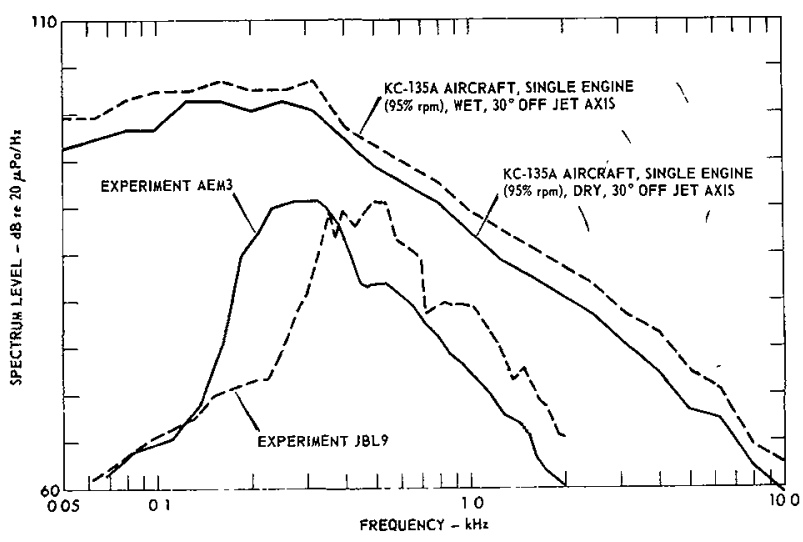

Fig. 6. - Comparison of jet noise spectra (KC-135A aircraft) with scaled spectra from Experiments JBL9 and AEM3. Distance $=76.2 \mathrm{~m}$.

Both sets of spectra are for a distance of $76.2 \mathrm{~m}$, i.E., $250 \mathrm{ft}$ (our data had to be extrapolated backward about $3 \mathrm{~m}$, a correction of $0.3 \mathrm{~dB}$ ). Before plotting our spectra, however, we had to scale them to make them comparable in frequency to the $\mathrm{KC}-135 \mathrm{~A}$ spectra. The scaling was done by invoking the amplitude-frequency product law that holds for nonlinear propagation distortion /4/. According to this law we may expect the same degree of nonlinear distortion at a given observation point if the spectrum is scaled down a decade in frequency while the level is scaled up $20 \mathrm{~dB}$. The two measured spectra treated in this way were taken from Experiments JBL9 $\left(O A S P L_{1 m}=145 \mathrm{~dB}\right)$ and AEM3 $\left(0 A S P L_{1 \mathrm{~m}}=142 \mathrm{~dB}\right)$.
Eiqure 6 shows that the level of the KC-135A noise is roughly $10 \mathrm{~dB}$ higher in the mid- and highfrequency regions than our noise. By demonstration our noise was definitely affected by nonlinear propagation distortion. The implication is that even stronger nonlinear effects were at work during the propagation of the KC-135A noise. Moreover, although the $K C-135 \mathrm{~A}$ is a very noise aircraft, many other current aircraft produce noise whose spectrum levels are higher than our scaled spectra. One therefore concludes that nonlinear effects are probably common in jet noise.

The conclusion of the study are as follows:

1. The noise used in this study is, after scaling, well within the jet noise range.

2. Nonlinear effects caused a strong generation of high-frequency noise.

3. The high-frequency generation occured both in the nearfield and in the farfield.

4. The growth in the nearfield exceeded that due to diffraction.

5. There was little or no generation of low-frequency noise.

6. At no measurement distance was small-signal behavior established for the high-frequency noise.

Acknowledgements. - This work was sponsored by the National Aeronautics and Space Administration, Office of Naval Research, Air Force Office of Scientific Research, and National Oceanic and Atmospheric Administration. The authors express their thanks to J.N. Cole, Aerospace Medical Research Laboratory, W-PAFB, Ohio, who Furnished the KC-135A data. 


\section{REFERENCES}

/1/ Theobald, M.A., "Experimental Study of Outdoor Propagation of Spherically Spreading Acoustic Waves of finite Amplitude," Technical Report ARL-TR-77-5, Applied Research Laboratories, The University of Texas at Austin, 1977 (ADA 039020).

12/ Webster, D.A., "Saturation of Plane Acoustic Waves and Notes on the Propagation of FiniteAmplitude Spherical Waves", Technical Report ARL-TR 77-4, Applied Research Laboratories, The University of Texas at Austin, 1977 (ADA 035694).

/3/ Webster, D.A., Theobald, M.A., and Blackstock, D.T., 9th International Congr. Acoust., 1977, II (Paper N32), 740.

14/ Webster, D.A., and Blackstock, D.T., "experimental Investigation of Outdoor Propagation of Finite-Amplitude Noise", NASA Rep. NASA CR-2992, 1978.

15/ Pernet, D.F., and Payne, R.C., J. Sound Vib., $1971,17,383$.

16/ Pestorius, F.M., and Blackstock, D.T., in Finite-Amplitude wave effects in fluids, Proceedings of the 1973 Symposium, Copenhagen (edited by Bjornø, L., ,) IPC Science and Technology Press, Ltd., Guildford, England, 1974, 24.

17/ Rudenko, 0.V., and Soluyan, S.I., Theoretical Foundations of Nontinear Acoustics, Nauka Press Moscow, 1975. English translation by Beyer, R.T., Plenum Pub7. Corp., New York, 1977, Chapter 10.

18/ Naugol'nykh, K.A., and Rybak, S.A., Zh. Eksp. Teor. Fiz., 1975, 68, 78 (Sov. Phys.-JEPT, 1975 $41,39)$.

19/ American National Standard Method For Circulation of the Absorption of Sound by the Atmosphere, ASA Standard S1.23, 1978,

(Proposed ANSI Standard S1.26). 